\title{
Rotational Velocities of "Field" Be Stars
}

\author{
J. Zorec ${ }^{1}$, R. Levenhagen ${ }^{2}$, J. Chauville $^{3}$, F. Royer ${ }^{4}$, N.V. Leister ${ }^{2}$, Y. \\ Frémat $^{3}$, and D. Ballereau ${ }^{3}$ \\ ${ }^{1}$ Institut d'Astrophysique de Paris, UMR7095/CNRS, 98 $8^{\text {bis }}$ bd. Arago, \\ F-75014 Paris, France (zorec@iap.fr) \\ ${ }^{2}$ Instituto de Astronomia, Geofisica e Ciencias Atmosfericas, Sao Paulo, \\ Brazil \\ ${ }^{3}$ Observatoire de Paris-Meudon, GEPI, FRE/2459, 92195 Meudon, \\ France \\ ${ }^{4}$ Observatoire de Genève, 1290 Sauverny, Switzerland
}

\begin{abstract}
Distributions of true rotational velocities as a function of spectral type for different luminosity class groups of B and Be stars are compared. While the distributions for B stars differ clearly from one luminosity class to another, those of Be stars differ less, which indicates that due to rotational effects many Be stars are considered more evolved than they actually are. Be stars 'select' a narrow range of true rotational velocities to display the Be phenomenon and those of latter spectral types rotate only marginally faster than the early ones.
\end{abstract}

\section{Stellar samples and rotational velocity distributions}

New spectra with resolutions from $R=15000$ to 40000 for nearly 1000 Be stars have already been obtained in the OHP (France), ESO (Chile) and LNA (Brazil) observatories. In this contribution we present the results based on the $v \sin i$ derived for the first 530 studied Be stars. We used here also the $v \sin i$ parameters of nearly $2000 \mathrm{~B}$ stars without emission (encompassing $\beta$ Cep, SPB, Bp, Bn and other B 'normal' stars) extracted from the CDS compilations (Globocki et al. 2000). B stars with and without emission lines were divided into three luminosity class groups: dwarfs (luminosity class V), subgiants (luminosity classes IV-V and V) and giants (luminosity classes II-III, III and III-IV). All $v \sin i$ distributions were corrected for observational errors and transformed into distributions of true rotational velocities with Lucy's (1974) algorithm, assuming random orientation of rotational axis.

\section{Results}

In Figs. $1 \mathrm{a}$ and $1 \mathrm{~b}$ are compared the true rotational velocities $V$ of $\mathrm{B}$ and $\mathrm{Be}$ stars. Be stars rotate from 1.5 to 2.5 times faster than B stars without emission. The increase of true rotational velocities among late B stars without emission is more pronounced than for Be stars. The separation of $V$ curves against spectral type at different luminosity classes is better defined for B than for Be stars, which 

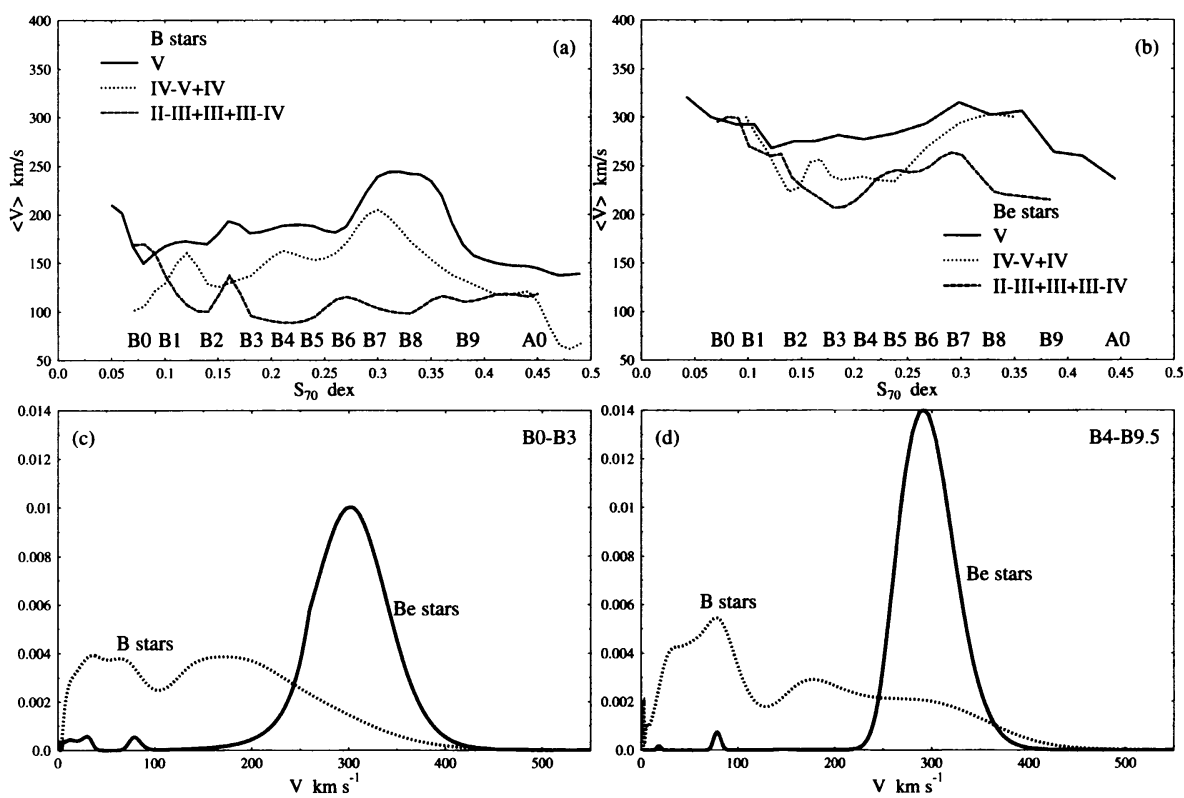

Figure 1. True rotational velocities against spectral type for different luminosity class groups of B (a) and Be (b) stars respectively. Normalized frequency distributions of true rotational velocities of early type B and Be stars (c) and late spectral types (d) of all luminosity classes

indicates that due to rotational effects many Be stars are probably considered more evolved than they actually are. The $V$ distributions of B and Be stars of all luminosity classes from V to III for two spectral type groups are compared in Figures $1 \mathrm{c}$ and $\mathrm{d}$. While the distributions for B stars are wider and irregular, due perhaps to the mixing of stars with different physical charactersitics, those for Be stars reveal a clear choice for narrow ranges of $V$. Late Be stars rotate marginally faster than early ones, as previously found by many authors (cf. Yudin 2001 and references therein). The maxima of $V$ distributions of Be stars and their dispersions can be somewhat larger than those shown in Figs 1c,d, because of a "saturation" effect on the spectral line widths produced by the gravitational darkening effect (Stoeckley 1968, Owocki 2003). The $v \sin i$ values tend thus to be underestimated for the fastest rotators.

\section{References}

Globocki, R., Gnacinski, P., Stawikowski, A. 2000, Acta Astron. 50, 509

Lucy, L.B. 1974, AJ 79, 749

Owocki, S.P. 2003, In: Stellar Rotation, IAU Symp. No 215, (eds.) A. Maeder, \& P. Eenens, ASP Confer. Ser., this volume

Stoeckley, T.R. 1968, MNRAS 140, 141

Yudin, R.V. 2001, A\&A 368, 912 